\title{
Strates
}

STRATES Matériaux pour la recherche en sciences sociales

$15 \mid 2008$

Union européenne - Voisinages. La quête d'une intégration régionale

\section{La rive sud a-t-elle besoin d'argent pour contribuer à l'espace financier euroméditerranéen ? Le cas des remises migratoires Europe-Maghreb}

Guillaume Almeras et Abderrahmane Hadj Nacer

\section{(2) OpenEdition}

Édition électronique

URL : http://journals.openedition.org/strates/6538

DOI : 10.4000/strates.6538

ISSN : 1777-5442

Éditeur

Laboratoire Ladyss

Édition imprimée

Date de publication : 1 janvier 2008

Pagination : 107-117

ISSN : 0768-8067

Référence électronique

Guillaume Almeras et Abderrahmane Hadj Nacer, "La rive sud a-t-elle besoin d'argent pour contribuer à l'espace financier euroméditerranéen ? Le cas des remises migratoires Europe-Maghreb», Strates [En ligne], 15 | 2008, mis en ligne le 22 janvier 2013, consulté le 08 septembre 2020. URL : http:// journals.openedition.org/strates/6538; DOI : https://doi.org/10.4000/strates.6538

Ce document a été généré automatiquement le 8 septembre 2020

Tous droits réservés 


\title{
La rive sud a-t-elle besoin d'argent pour contribuer à l'espace financier euroméditerranéen? Le cas des remises migratoires Europe- Maghreb
}

\author{
Guillaume Almeras et Abderrahmane Hadj Nacer
}

\section{NOTE DE L'AUTEUR}

Cet article est tiré du rapport remis à l'Institut de Prospective Économique du Monde méditerranéen fin 2006.

La constitution d'un espace financier euroméditerranéen est une priorité pour une région intégrée économiquement. Pour le moment, l'Union européenne privilégie la solution d'une "BERD-Med", sur le modèle de la Banque Européenne pour la Reconstruction et le Développement qui a été un des instruments de la modernisation économique de l'Europe de l'Est et la reconnexion Est-Ouest du continent. Cet article défend l'idée qu'une telle approche n'est pas la seule possible pour la Méditerranée voire qu'elle n'est peut-être pas nécessaire. Car au contraire de l'Europe de l'Est, la rive sud de la Méditerranée n'a pas besoin d'argent. Cette idée est difficile à admettre dans les logiques classiques d'aide au développement. Mais les seuls transferts migratoires de l'Europe vers le Maghreb constituent une manne considérable. À l'heure où l'on tente de trouver un nouveau souffle au Protocole de Barcelone, ce phénomène des remises migratoires reste largement ignoré, à plusieurs titres : en lui-même car, pour les trois pays du Maghreb (Maroc-Algérie-Tunisie), chiffres et données sont incertains (première partie); pour ce qu'il signifie quant au comportement des populations maghrébines installées et travaillant en Europe (deuxième partie); pour ce qu'il 
représente comme levier financier de développement (troisième partie) et, au-delà, comme nouvelle manière d'envisager les rapports méditerranéens (quatrième partie).

Pour valoriser l'épargne en général et en particulier celle des personnes qui vivent désormais à cheval sur les deux rives, le Maghreb a, avant tout, besoin de moderniser le fonctionnement et les services de ses banques commerciales. Tel nous parait être le fil directeur de la construction d'un véritable espace financier euroméditerranéen. Cet article en donne les orientations principales, sans s'attacher aux différences qui, bien sûr, distinguent le Maroc de l'Algérie ou de la Tunisie.

\section{Les transferts issus de l'émigration maghrébine en Europe restent méconnus}

3 En termes quantitatifs, la problématique des transferts issus des résidents maghrébins à l'étranger demeure difficile à cerner, cela tant dans les pays d'accueil (la France ne produit ainsi aucune statistique officielle précise), que dans les pays d'origine. Aucune structure propre n'a été mise en place pour recueillir ces fonds en Algérie. En Tunisie, les banques publiques ne distinguent pas dans leurs livres les dépôts selon leur origine. Même au Maroc, sans doute l'un des pays les mieux organisés au monde en ce domaine, ce n'est que récemment que l'importance du phénomène à l'échelle nationale a été pleinement reconnue, avec la création d'un ministère des Marocains résidents à l'étranger - les remises représentant la première source de devises du Royaume.

4 Comment expliquer un tel désintérêt? Il faut croire que ces flux issus de l'émigration gênent tant les pays d'accueil que les pays d'origine - comme s'ils avaient quelque chose d'un peu honteux, que ce soit pour les pays émetteurs (le thème des immigrés qui font sortir des devises) que pour les pays récepteurs (le thème de la dépendance à l'égard de l'étranger). On ne peut comprendre autrement, en effet, qu'une telle manne ait à ce point été délaissée jusqu'à aujourd'hui et demeure si mal cernée. De plus, on a longtemps cru que ces transferts étaient voués à se tarir, au fur et à mesure que les migrants abandonnaient l'idée d'un retour au pays. Pourtant, depuis cinq ans, les sommes transférées croissent et de manière importante. Au Maroc par exemple, les transferts migratoires ont augmenté au premier semestre 2006 de $16 \%$ par rapport à la même période l'année précédente, faisant suite à quinze années de hausse soutenue et pratiquement ininterrompue.

5 Il faut dire que tout se passe comme si les pays du Maghreb avaient constitué leurs classes moyennes hors de leurs frontières, et notamment en Europe. Cela oblige à considérer d'un œil nouveau le comportement des populations migrantes et de celles nées en Europe, à la recherche d'une intégration qui n'est en réalité réalisée ni vraiment dans le pays d'accueil ni dans le pays d'origine mais bien à cheval entre les deux.

De cette situation résultent d'énormes flux financiers transméditerranéens. Les chiffres disponibles sont essentiellement ceux du Maroc : de 3 à 4 milliards d'euros de transferts migratoires par an depuis cinq ans, dont plus de la moitié vient de France. Ces transferts représentent $22 \%$ des recettes courantes de la balance des paiements marocaine, plus de $50 \%$ des recettes d'exportation, ou encore un revenu supérieur à celui du tourisme ou des phosphates. Avec toutes les réserves qu'appellent les évaluations en ce domaine, il faut parler, au minimum, d'un flux annuel de 5 à 10 
milliards d'euros entre l'Europe et les trois pays du Maghreb. Un flux à comparer au total des investissements directs venus d'Europe et réalisés au cours des six dernières années, dans les trois pays du Maghreb : 4,5 milliards de dollars (pour 19 milliards à l'adresse des Peco). À comparer encore aux aides internationales (crédits remboursables) que reçoit un pays comme le Maroc chaque année : 320 millions d'euros de l'Europe ${ }^{1}, 189$ millions de dollars de la Banque mondiale/IFC et 164 millions de la Banque africaine de développement.

7 Le montant des transferts annuels marocains est désormais comparable à celui réalisé par la communauté portugaise en France (3,8 milliards d'euros en moyenne par année). Cela montre que les montants de transfert ne baissent pas forcément avec l'installation durable des populations migrantes dans les pays d'accueil. Bien organisés, ces flux peuvent même augmenter: en France, la banque portugaise Borges Irmao a vu son montant de collecte multiplié par six entre 1980 et 1990, en pleine phase d'enracinement des populations portugaises.

Mieux, cela permet d'envisager, à rebours de ce qu'on imagine généralement, que le développement économique des pays du Maghreb puisse non pas tarir mais bien augmenter les transferts, en même temps qu'il contribuerait à fixer l'épargne constituée localement. Car, en matière de flux financiers Sud-Nord cette fois, il faut tenir compte d'une masse considérable d'environ 7 milliards d'euros par an ayant pour origine les pays du Maghreb et ne trouvant à s'investir qu'en Europe ou aux États-Unis, faute de trouver localement des supports adéquats de placement: fortunes privées, avoirs en devises des banques et des entreprises délibérément laissés dans les livres des banques internationales et allant gonfler les réserves de changes nationales conservées auprès de banques américaines et européennes... Alors que les transferts sont la première source de devises pour le Maghreb, en sens contraire l'épargne maghrébine fuit littéralement la région par manque d'opportunités et manque de confiance.

L'enjeu financier du développement du Maghreb, s'éclaire donc: il n'a pas besoin d'argent, car il en a. Il a surtout besoin de conditions fiables de bancarisation et d'investissement local. Cette perception différente de la confiance explique en large partie que les immigrés portugais installés en France soient plus nombreux à manifester leur volonté de retour au pays que les immigrés marocains, à ancienneté égale des deux courants migratoires. On comprend donc les trois impératifs pour mieux valoriser le rôle des remises migratoires dans l'espace financier euroméditerranéen : confiance, stabilité, allocation productive des fonds.

\section{Migrants : des attentes nouvelles donc des produits financiers à adapter}

\section{Du soutien familial au placement et à l'investissement}

Dans les transferts issus de l'émigration, la part du soutien familial demeure prépondérante, comme en témoigne le fait que, pour le Maroc, le montant des envois varie à proportion de la qualité des campagnes agricoles. Sur place, les bénéficiaires directs des transferts emploient encore l'argent essentiellement pour les dépenses de consommation courante (51\%), bien avant l'éducation et la santé (18\%) et le logement (14\%). La part des investissements est néanmoins de plus en plus importante. Elle atteindrait désormais $18 \%$ des transferts tunisiens. Les fonds mobilisés à ce titre sont 
essentiellement investis dans l'immobilier ( $83 \%)$, le commerce (12\%), l'industrie (3\%) et l'agriculture (2\%).

C'est cette nouvelle orientation des transferts vers l'investissement qui permet de comprendre pourquoi les transferts issus des populations migrantes maghrébines croissent. Des populations durablement installées en Europe ont compris qu'elles avaient tout intérêt à profiter du différentiel de pouvoir d'achat des deux côtés de la Méditerranée - à faire fructifier là-bas l'argent gagné ici. Car les transferts sont de plus en plus le fait des populations nées en Europe, ne connaissant pas forcément le pays d'origine de leurs ascendants ou n'en ayant pas une image très flatteuse - n'y envisageant en tout cas aucune installation définitive pour la très grande majorité.

Cela signifie deux choses. D'abord, que les populations maghrébines installées en Europe n'ont pas véritablement intérêt à s'y intégrer uniquement mais au contraire à cultiver leur double appartenance, quitte à afficher de manière plus vive leurs particularismes - c'est là certainement une piste pour comprendre la diffusion récente $\mathrm{du}$ port du voile, qui est d'abord un phénomène touchant les femmes jeunes. Paradoxalement, les générations européennes de l'immigration maghrébines pourraient bien être moins intégrées dans leur pays d'accueil que leurs parents. Le phénomène se constate également de la part des populations turques en Allemagne. Financièrement ensuite, cela confirme que les transferts ne vont pas décroître mais qu'ils vont changer d'objet: non plus le soutien familial mais les opportunités de placement. Un signal fort est à cet égard l'importante récente souscription du capital de Maroc Telecom par des non-résidents.

13 Au total, dans les trois pays du Maghreb, un flux régulier et important de dépôts recherche désormais de plus en plus une épargne liquide, de plus en plus attirée par des opportunités de placement; une épargne de précaution (santé, retraite); une épargne de projet (immobilier, investissements). Ces demandes sont actuellement loin d'être remplies pour les migrants. En France aujourd'hui, il n'existe par exemple aucun support d'épargne simple, accessible à tous et sécurisé, qui favorise l'investissement immobilier au Maghreb ou le placement dans le cadre de privatisations. Et elles sont encore moins remplies pour les nationaux. À ce titre, la question de la mobilisation de l'épargne, des deux côtés de la Méditerranée, parait bien être une des principales clés du développement financier - du développement tout court - des pays maghrébins.

\section{De nouveaux comportements à cheval sur les deux rives de la Méditerranée}

Quoique peu souligné, l'alignement des évolutions sociales des deux côtés de la Méditerranée est bien réel : la France et le Maroc ont connu des affaires de «foulard islamique ", de manière différente, certes, mais au même moment. L'alignement accéléré est également frappant dans le domaine des comportements démographiques : l'accroissement annuel du Maroc a chuté de 2,1\% (1982-1994) à 1,4\% aujourd'hui, le taux de fécondité est passé de 5,5 enfants en 1982 à 2,5 en 2002 - des taux à l'européenne. Un nouveau mode d'intégration des populations maghrébines se dessine ainsi, qui intervient à une échelle transnationale, même si les revendications identitaires sont de plus en plus vives à l'échelle nationale. Les particularismes affichés, notamment religieux, ne doivent pas conduire à négliger la proximité culturelle du Maghreb par rapport à l'Europe en général et à la France surtout. On ne peut oublier 
qu'en fait de programmes télévisés, les journaux maghrébins donnent ceux des chaînes françaises... Au-delà des particularismes culturels et nationaux, une région socioéconomique commune émerge des deux côtés de la Méditerranée, marquée par une porosité accrue des flux, portée par la complémentarité forte créée par des niveaux de développement nettement distincts.

D'un point de vue macroéconomique en effet, la complémentarité tient aux déséquilibres démographiques: le vieillissement relatif des populations européennes devrait provoquer une migration des stocks d'épargne du Nord au Sud d'un côté et le retard de niveau de vie et la jeunesse relative des populations du Sud de l'autre devraient y entretenir un appétit de consommation et porter une croissance que le Nord, sans doute, ne connaîtra plus. Ainsi, l'épargne et le crédit sont bien les clés d'un développement maghrébin qui appelle désormais des solutions globales, c'est-à-dire méditerranéennes.

D'ores et déjà, pour ce qui concerne les flux financiers, il ne faudrait donc plus raisonner en termes d'immigration mais s'intéresser plutôt aux personnes physiques et morales dont les dépenses d'investissement et de consommation sont largement et durablement réalisées d'une rive à l'autre. Il s'agit de penser l'intégration des populations migrantes non plus de manière nationale, unilatérale mais participant à un espace méditerranéen de flux.

17 En d'autres termes, le développement économique des pays du Maghreb ne dépend nullement de l'aide financière européenne - y compris pour la réalisation d'infrastructures à l'échelon national ou régional. La question concerne bien davantage la mobilisation et la collecte de l'épargne existante, au Sud comme au Nord, et sa réallocation productive.

\section{Les implications pour le secteur bancaire maghrébin}

\section{Faire de la mobilisation de l'épargne une priorité stratégique}

La réallocation productive des flux financiers issus de l'immigration maghrébine est actuellement compromise à un double titre : d'abord les flux disparaissent en large partie dans des canaux informels, faute d'être mieux organisés ; ensuite, ils ne trouvent guère de supports d'épargne adéquats. Par ailleurs, l'épargne constituée localement fuit la région.

19 À partir de 2000, le solde extérieur courant des économies émergentes est devenu excédentaire. Il a atteint 423 milliards de dollars en 2005. Cela signifie qu'à rebours des schémas classiques de l'économie du développement, les pays émergents génèrent des excédents d'épargne qu'ils placent dans les économies développées. Autrement dit, le Sud finance le Nord. Ce phénomène est essentiellement le fait de la Chine (158 milliards de dollars en 2005) et du Moyen Orient (196 milliards). Au sein du Maghreb, seule l'Algérie participe de ce phénomène, du fait du renchérissement des produits pétroliers. Pour le reste, la région est très loin d'enregistrer les excédents commerciaux chinois. Et pourtant, nous l'avons souligné, l'épargne maghrébine se place elle aussi largement dans les pays développés - seul son caractère informel dissimule le phénomène. 
20 Ici comme ailleurs, une véritable pénurie d'investissements se constate, du fait de la rareté des projets véritablement innovants et des difficultés de tous ordres que rencontrent ceux qui les portent, du fait d'un rendement insuffisant des capitaux immobilisés. Cette situation est inquiétante si l'on songe que le bassin méditerranéen est peut-être la principale zone de contacts, fractures et interdépendance de la planète. $\mathrm{Au}$ Maghreb, de violents déséquilibres pourraient ainsi apparaître à terme, avec quelques îlots privilégiés destinés aux Européens (tourisme, retraite et santé), et des territoires assumant une fonction d'arrière-cour pour l'Europe du sud qui y délocaliserait ses industries polluantes et ses activités à bas salaires ${ }^{2}$. Prévenir la réalisation d'un tel scénario obligerait à rompre avec deux présupposés qui ont jusqu'ici orienté l'aide européenne. Le premier est que le développement repose d'abord sur l'investissement et notamment l'investissement extérieur, alors qu'en réalité il repose d'abord sur la mobilisation de l'épargne qu'on a pourtant largement négligée. Le second est que chacun des trois pays du Maghreb justifierait de dispositifs particuliers, alors qu'il faut absolument raisonner à l'échelle du Maghreb - et qu'en fait, le Maghreb même commençant à devenir étroit à l'échelle internationale, il faudra bien en venir à penser « Méditerranée ».

\section{Des systèmes bancaires qui contraignent le développement plus qu'ils ne le servent}

21 Lorsqu'on pense solutions de développement, d'un point de vue institutionnel, on invoque inévitablement l'apport, sous forme d'aides et de concours remboursables, de capitaux longs. On envisage le financement de grandes infrastructures, ainsi que le soutien des entreprises innovantes à travers des fonds de capital-risque ; à ces mesures s'ajoute, depuis peu, le soutien au microcrédit. On oublie ainsi généralement l'essentiel : le rôle des banques commerciales tout simplement, sur le terrain, dans la sélection des projets, comme dans la maîtrise des moyens de paiement.

De fait, ce n'est pas le manque de ressources ou de moyens qui contraint le développement des pays du Maghreb, mais, au niveau des banques, le poids de pratiques et d'usages qui étranglent le développement. Une situation que les banques ne créent pas sans doute, qu'elles subissent même, mais qu'elles répercutent à l'ensemble des agents économiques et particulièrement aux PME. Au Maghreb comme ailleurs, en Chine notamment, seul le rôle de levier du système bancaire, seul l'effet multiplicateur du crédit, peuvent être déterminants. Cela suppose de mobiliser une épargne qui est aujourd'hui peu productive et gérée au niveau individuel des entreprises et des particuliers, et de prendre des risques.

Or les systèmes bancaires actuels sont les pendants d'économies nationales de rente, fondées sur l'exploitation de matières premières et de ressources foncières ou paysagères, sur la recherche de profits spéculatifs ou commerciaux à court terme, bien plus que sur de véritables stratégies de développement. De là des marchés financiers étroits, sur lesquels les positions qu'occupent les banques étrangères, particulièrement françaises, ne sauraient être présentés comme d'éclatants succès... Parce qu'elles sont encore des conservateurs de valeurs avant tout et opèrent sur des marchés limités à des fractions privilégiées de particuliers et d'entreprises, parce qu'elles accumulent de nombreux impayés et défaillances, parce qu'elles sont fragilisées par un difficile traitement des contentieux (procédures judiciaires longues, coûteuses, à l'issue 
incertaine, faibles incitations fiscales au provisionnement des créances en souffrance), les banques maghrébines limitent l'offre de crédit en quantité (beaucoup d'entreprises n'y accèdent pas), en qualité (peu d'engagements à moyen-long terme), ainsi qu'en termes de conditions (garantie patrimoniale impérative, cash collatéral exigé pour les opérations de commerce international). Au total, le crédit n'est pas facile et il est cher. Dans les trois pays, les systèmes bancaires contraignent le développement économique bien plus qu'ils ne le servent.

Comme dans beaucoup d'autres pays en développement, les banques maghrébines sont structurellement sur-liquides, mais leurs ressources étant absorbées par les titres d'État à court terme ou bien nourrissant d'importants crédits à des entreprises publiques mauvaises payeuses, ces banques disposent finalement de liquidités insuffisantes pour supporter le développement économique. Pour capter les dépôts des particuliers, elles se livrent à une véritable surenchère en termes d'ouverture d'agences, en Tunisie comme au Maroc. Peu est fait, cependant, pour transformer ces dépôts en une épargne de long terme, mobilisable pour l'investissement.

\section{Le Maghreb comme pôle économique unifié}

25 Avec le retour à la stabilité politique de l'Algérie, le Maghreb est récemment devenu un pôle attractif pour les entreprises françaises, dont l'intérêt se porte en effet désormais vers la région dans son ensemble et non plus seulement vers l'un ou l'autre des trois pays. Pourtant, comme espace économique unifié intégrant les trois pays, le Maghreb n'existe pas! Et à moyen terme il y a peu de chances qu'une initiative politique commune aux trois pays le crée. Il faut cependant tenir compte d'une impulsion extérieure et être prêt à raisonner dans le cadre d'une zone de co-développement euroméditerranéenne, marquée par l'accroissement et la régularité des flux humains, commerciaux et financiers. Ceux-ci donneront certainement un caractère toujours plus homogène à la région, au-delà des appartenances nationales.

Alors que leurs flux d'affaires vont sans doute se renforcer avec l'UE et au-delà (ÉtatsUnis, Chine), force sera pour les trois pays de se rapprocher, en effet, dans la mesure où aucun d'eux n'a la taille critique à l'échelle internationale. Les investisseurs internationaux développeront des stratégies communes, tendront à ne retenir qu'une seule implantation pour couvrir la zone. De même les flux entre pays du Sud devraient croître ; en 2006 déjà, un million de touristes algériens auront visité la Tunisie ; l'Égypte investit déjà plus que la France dans la région particulièrement dans les télécoms, etc. On peut donc parier sur la constitution d'un marché maghrébin de plus en plus homogène, même s'il n'est pas accompagné du libre-échange ni d'une communauté politique.

Il n'y a sans doute actuellement aucune volonté chez les dirigeants des trois pays de faire rapidement éclore un Maghreb économiquement unifié. Pourtant, celui-ci se fera. Mais de manière exorbitée, à travers l'arrimage commercial, industriel et financier sans cesse accru avec la rive nord de la Méditerranée. Et ce scénario, s'il se réalise, ne se fera sans doute pas sans clivages : quant au développement (un Maghreb à deux vitesses) et quant à sa diffusion (des pôles tournés vers l'international, d'autres prenant peut-être une dimension régionale et d'autres encore ayant de grandes difficultés à s'affranchir des pesanteurs locales). Un Maghreb de plus en plus homogène à l'échelle 
internationale, pourrait donc aussi bien être morcelé à l'échelle locale, faute d'actions d'ensemble et d'envergure favorisant le développement entre les trois pays.

\section{Rompre avec les schémas classiques de l'aide au développement et de l'immigration}

On voit donc que la constitution d'un espace financier euroméditerranéen passe par un renouvellement des cadres conceptuels du développement au Nord et au Sud. C'est dans cette optique nouvelle qu'on peut esquisser ce qui pourrait être une institution financière méditerranéenne.

\section{Créer une institution financière multilatérale mobilisant l'épargne comme un levier}

Dans les transferts issus de l'émigration, nous l'avons dit, la part correspondant au soutien familial demeure importante mais d'autres mobiles apparaissent, liés à la recherche de placements, pour profiter du différentiel de pouvoir d'achat des deux côtés de la Méditerranée. Or le manque actuel d'opportunités de placement provoque dans les trois pays une spéculation sur l'immobilier aux effets délétères (éviction des PME des centres urbains d'activité, abandon des programmes de logements sociaux), en même temps qu'il provoque l'évasion d'une grande partie de l'épargne constituée localement. Seul l'investissement productif peut pallier ce manque général de profitabilité économique, mais son développement se heurte à la rareté de ressources longues. De sorte que la seule manière de rompre ce cercle vicieux est d'agir sur la collecte et la transformation de l'épargne, avec pour fer de lance les transferts issus de l'émigration. Ce qui manque, c'est un opérateur international capable de gérer une telle transformation.

Il s'agirait ainsi :

31 - d'organiser et de faciliter la collecte de cette épargne, de part et d'autre des deux rives de la Méditerranée (ce qui suppose des accords entre les différents États sur la convertibilité euro/monnaie locale). Les transferts devraient être mobilisés sous forme d'épargne à travers des livrets ciblés, dont la convertibilité et la rémunération pourraient être garanties et la fiscalité aménagée : livrets d'épargne liquide, d'épargne immobilière, d'épargne santé et retraite, livrets d'investissement (développement industriel, privatisations...);

- de sécuriser la gestion, la conservation et le réemploi de cette épargne : une mission de service public devant être confiée à une institution multilatérale, qui serait la seule signature « triple $\mathrm{A}$ » de la région ;

- d'assurer le réemploi productif de cette épargne en apportant une expertise de sélection et de financement de projets.

Une fois les livrets d'épargne définis par accord entre États, la collecte serait directement assurée par les banques commerciales privées, des deux côtés de la Méditerranée, dans le cadre donc d'un partenariat public-privé. Les fonds seraient confiés en tout ou partie à l'institution multilatérale, les banques commerciales étant rémunérées pour la collecte. À cinq ans, la gestion d'un encours de 10 milliards d'euros peut raisonnablement être envisagée. Ces fonds auraient deux emplois principaux : ils 
financeraient des missions d'investissement d'intérêt général de long terme (aménagement des territoires, logement social, grands équipements collectifs) et ils permettraient la mise en place d'un dispositif de garantie de prêts bancaires permettant de faciliter l'accès au crédit des PME et des particuliers pour certaines finalités précises (création d'entreprise, équipement professionnel, nouvelles technologies, accession à la propriété...).

Sur le plan de la gouvernance, l'institution devrait, comme tout établissement financier, répondre de sa solvabilité et de ses résultats sur ses fonds propres. Son actionnariat pourrait être ouvert à des investisseurs privés. L'enjeu serait d'aller vers une gouvernance de plus en plus multilatérale, méditerranéenne et paritaire Nord/sud. Au total, il s'agirait de la solution la moins onéreuse d'aide au développement puisque, s'appuyant sur la mobilisation sous forme d'épargne des flux monétaires issus de l'immigration, elle ne demanderait pas un euro de subvention. Cette institution financière n'en serait pas moins une institution financière de référence et une autorité de paiement incontournable, tant pour les entreprises désireuses d'investir localement, que pour les programmes d'aide internationaux.

\section{Changer notre vision du développement de la rive sud de la Méditerranée et de ses émigrés}

Pour favoriser le développement euroméditerranéen, il faut beaucoup d'argent et l'apport de ressources longues. Il n'est pourtant besoin ni d'aides ni de subventions, là est la nouveauté essentielle dans l'approche du financement du développement. La solution, ni totalement publique ni totalement privée, est précisément à la rencontre d'une mission d'intérêt général et de l'activité courante des établissements financiers privés. Il serait en effet possible d'assurer la réallocation productive des flux financiers qui transitent régulièrement entre les deux rives de la Méditerranée et de s'en servir comme levier, en marquant ainsi l'existence d'une zone de co-développement qui n'attend que d'être reconnue pour telle.

Une telle initiative dérogerait sans doute aux schémas classiques de l'aide au développement. Ne serait-ce que parce qu'elle associerait étroitement, dans sa mission même, une institution publique et des établissements privés. Et parce qu'elle valoriserait les modes de vie à cheval sur les deux rives de la Méditerranée.

Ces trente dernières années, on ne peut pas dire du Maroc et encore moins de l'Algérie qu'ils se sont développés à la hauteur de leur potentiel et de leurs ressources. Ces deux pays - et la Tunisie également, dans une moindre mesure - ont souffert non pas d'un manque mais d'une allocation inadéquate de ressources. Des franges importantes de leurs populations, en situation précaire, ont dû tenter leur chance ailleurs. Au cours de la même période, l'Europe en général et la France en particulier ont absorbé un grand afflux de populations immigrées dans un contexte de croissance plutôt molle, ponctué de crises économiques tout en supportant un chômage endémique. Dans un tel contexte, les populations immigrées auront le plus souvent dû se contenter de métiers peu rémunérateurs et auront même souvent vécu dans un état de quasi-pauvreté, tout en transférant, malgré tout, des sommes importantes dans leurs pays d'origine. Des sommes qui ne se sont pas taries avec l'installation durable de ces populations en Europe et l'accès, pour certaines, au statut des classes moyennes. De là un véritable circuit économique de croissance au sein duquel tout le monde devait être gagnant : les 
pays d'origine de par les sommes transférées par leurs migrants, et le pays d'accueil vers lequel l'argent ne pouvait que revenir en large partie, quand on connaît le poids de la France dans les échanges commerciaux de trois pays qui importent la quasi-totalité de leurs biens manufacturés. Bien que peu de chiffres soient disponibles à cet égard, il n'est donc pas aberrant de retenir l'hypothèse que l'immigration maghrébine en Europe et notamment en France aura généré une croissance suffisante pour se financer!

C'est là une façon de reconnaître que l'Europe et la France sont déjà engagées avec le Maghreb dans un processus de développement durable commun, qui demande seulement à être vraiment organisé.

\section{BIBLIOGRAPHIE}

Banque mondiale, 2005, Global Economic Prospects 2006, Economic Implications of Remittances and Migration, Washington.

BEI 2006, Étude sur les transferts de fonds des migrants méditerranéens d'Europe, Femip, polygr.

Charbit Y., Chort I., 2006, « Les transferts monétaires des migrants : pays industrialisés et pays en développement », Revue Européenne des Migrations Internationales, Volume 22, nº 2, p. 127-154. Accessible en ligne : http://remi.revues.org/document2825.html

Ozden C., Schif M. (dir.), 2005, International Migration, Remittances, and the Brain Drain, Washington, Palgrave Macmillan, Banque mondiale.

OCDE, 2005, Migration, transferts de fonds et développement, Paris.

Straubhaar Th., Vadean F., 2006, « Les transferts de fonds internationaux des émigrés et leur rôle dans le développement ", Institut d'économie internationale de Hambourg (HWWA), in OCDE, Sopemi, Perspectives des Migrations Internationales, 2006, Paris.

\section{NOTES}

1. $46 \%$ des fonds Meda et $54 \%$ de la BEI.

2. Voir par exemple le développement des centres d'appels, dont le Maroc est le premier destinataire africain (150 centres d'appel à ce jour, 20000 employés). 


\section{RÉSUMÉS}

L'article rappelle que les transferts financiers issus de l'émigration maghrébine en Europe sont un phénomène relativement méconnu, alors qu'ils représentent une masse financière déterminante. Les migrants ont des attentes et des besoins nouveaux: les populations durablement installées en Europe ont compris qu'elles avaient tout intérêt à profiter du différentiel de pouvoir d'achat des deux côtés de la Méditerranée. Financièrement, cela signifie que les transferts ne vont pas décroître, au contraire, mais qu'ils vont changer d'objet : non plus le soutien familial mais les opportunités de placement. La mobilisation de l'épargne des deux côtés de la Méditerranée, paraît bien être une des principales clés du développement financier du développement tout court - des pays maghrébins. L'article dessine enfin les grandes lignes de ce qui devrait devenir une institution financière régionale euroméditerranéenne : une institution multilatérale publique, proposant un livret d'épargne Euromed; cette banque publique s'appuierait sur des consortiums Nord-Sud de banques privées, bénéficiant des garanties publiques pour délivrer les services bancaires modernes qu'attend l'importante épargne de la rive sud, pour l'instant sous employée et non orientée vers l'investissement productif.

The money transfers (remittances) coming from the North African emigrants working and residing in Europe are not a well known phenomena, although they reach considerable amounts. The international migrants have new expectations and needs. North African migrants who reside in Europe have understood that they should take advantage of the huge differences in the average standards of living between both banks of the Mediterranean. On a financial point of view, that means that the transfers are not likely to decrease. On the contrary, they will increase and their aim will change, with a shift from the traditional support of the purchase power of families to new investment opportunities. The capacity to collect the savings of the migrants seems to be one of the keys of the development in the countries of the Maghreb. The authors try to draw the mixture of what could become a Mediterranean financial institution. This public multilateral institution would provide Euromed saving accounts and would be backed by NothSouth consortiums of private banks. Its sustainability would be garanteed by public administrations. It would deliver modern banking services in order to collect the savings and to orient them toward productive investments.

\section{INDEX}

Index géographique : Europe, Méditerranée, Afrique du Nord

Mots-clés : espace financier, flux migratoires, système bancaire, développement économique

\section{AUTEURS}

\section{GUILLAUME ALMERAS}

Consultant spécialisé dans les questions financières

\section{ABDERRAHMANE HADJ NACER}

Ancien gouverneur de la banque centrale d'Algérie, banque d'affaires 